\title{
Revisiting Caloric Restriction as Therapeutic Strategy for Metabolic Syndrome, T2DM and Obesity
}

\author{
Vinod Nikhra* \\ Department of Medicine, Hindu Rao Hospital and NDMC Medical College, India \\ *Corresponding author: Vinod Nikhra, Senior Chief Medical Officer and Consultant, Department of Medicine, New Delhi, India
}

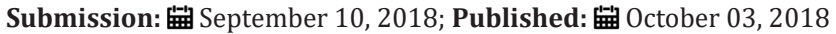

\begin{abstract}
Overnutrition and adiposity: Overnutrition contributes to chronic energy surplus leading to adiposity, insulin resistance (IR), metabolic syndrome (MetS) and obesity with its fallouts including increased oxidative stress, altered glucose, fat and protein metabolism, and altered skeletal muscle mitochondrial function.

Redox balance and thioredoxin system: The cellular redox balance is regulated by activity of several antioxidant systems including thioredoxin (TXN) system. Thioredoxin interacting protein (TXNIP) modulating the activity of TXN, is a key player in regulation of glucose homeostasis and lipid metabolism. The overexpression of TXNIP in T2DM, MetS and obese subjects is associated with metabolic abnormalities including apoptosis of $\beta$-cells, decreased insulin sensitivity and energy expenditure. The reduced oxidative capacity of skeletal muscle leads to accumulation of intramyocellular triglyceride (IMTG) and affects mitochondrial function. TXNIP influences metabolic regulation mainly through insulin release from $\beta$-cells, glucose production from liver and glucose uptake in peripheral tissues. In addition, it acts as a nutrient sensor in discrete regions of brain.
\end{abstract}

Effects of CR on metabolic homeostasis: Caloric restriction (CR) is a potentially effective therapeutic strategy to improve adiposity and insulin sensitivity and action at tissue level. CR associated weight loss decreases IMTG and improves mitochondrial function in skeletal myocytes. The decrease in adipose mass, oxidative stress and inflammation lead to downregulation of TXNIP, eliminating its inhibitory effect on glycolysis, glucose transporters, insulin receptors and receptor substrate, insulin-stimulated Akt activation and phosphatidylinositol 3-kinase (PI3K).

Conclusion-novel additives to polypharmacy: To endorse CR is of immense value, as it consistently leads to improved cardiometabolic outcomes and exerts beneficial effects on every organ system. Yet, CR is difficult to implement in practice for multiple reasons including dietary adherence and perceived decrease in QOL. Still, the focus on CR is important within a specific disease context to clearly delineate underlying mechanisms and exploit the research to achieve therapeutic goals. TXNIP is a potential therapeutic target. Certain anti-diabetic agents like metformin, GLP-1 agonists and CRMs like resveratrol have been shown to inhibit TXNIP expression. Verapamil-a calcium channel blocker, tranilast-a tryptophan metabolite and allopurinol reduce TXNIP levels in vivo and in vitro studies. Certain calcium channel blockers by lowering TXNIP levels protect $\beta$-cells from apoptosis. On a cautious note, the loss of TXNIP may have serious consequences as TXNIP expression is required for maintaining normal fasting glycaemia and TXNIP being a tumour suppressor, its loss is associated with increased incidence of cancer.

Keywords: Caloric restriction; Caloric restriction mimetics; Insulin resistance; Metabolic syndrome; Type 2 Diabetes mellitus; Obesity; Redox balance; Thioredoxin (TNX); Thioredoxin interacting protein (TXNIP)

\section{Introduction}

\section{Overnutrition and SALS}

Overnutrition is ingestion of food in excess causing a mismatch between actual caloric requirement and intake, which results in chronic energy surplus. In addition, the modern-day sedentary activity lifestyle (SALS) adds further to the imbalance between calorie intake and energy expenditure. The imbalance and chronic energy surplus leads to adiposity, which is accumulation of excess energy in adipose tissue as lipids. The adiposity contributes to reduced insulin sensitivity, metabolic syndrome (MetS) and obesity with its fallouts. The insulin resistance (IR) has been linked to increased oxidative stress, altered glucose, fat and protein metabolism, and altered skeletal muscle mitochondrial function [1].

The diet-gene interaction is an important determinant of health (Figure 1). The MetS encompassing abdominal adiposity, IR, dyslipidaemia and hypertension, accounts for a major public health threat both in developed as well as developing regions of the world. The rise in obesity and MetS prevalence in recent decades are attended by the background changes in dietary and lifestyle patterns, reflecting increased nutrient availability and consumption, and modern-day sedentary activity level of daily living. 


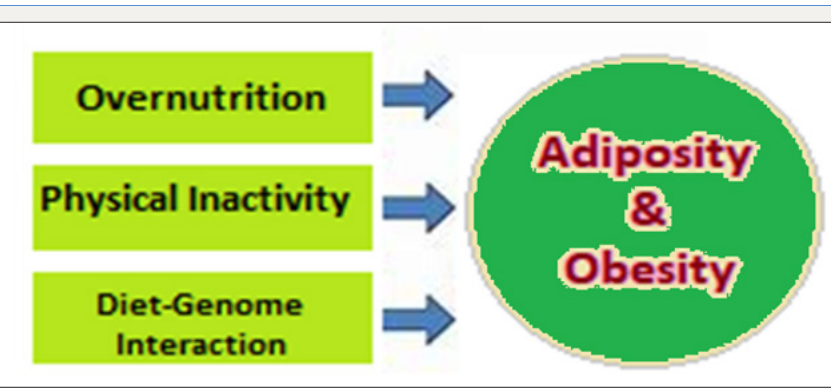

Figure 1: Adiposity: overnutrition, SALS and chronic energy surplus.

The pooling of positive energy balance, that is, food and energy intake greater than energy expenditure, leads to expansion of the white adipose tissue (WAT), occurring through both an increase in adipocytes volume as well as the development of new WAT transdifferentiated from brown adipocytes to store additional energy.

\section{Relevance of caloric restriction}

Consistent with the pathophysiological phenomenon leading to adiposity, MetS and obesity, reducing caloric intake can be considered a potentially effective therapeutic strategy to improve insulin sensitivity, reduce IR and adiposity. The underlying molecular and cellular mechanisms of improved insulin sensitivity with CR in skeletal muscle, however, remain to be fully understood [2]. The studies have documented that the CR enhances insulin sensitivity without improving mitochondrial function [3]. The Skeletal muscle accounts for a major part of insulin-stimulated glucose disposal and important tissue for peripheral glucose uptake. The IR leads to accumulation of diacylglycerol (DAG) and ceramide in skeletal muscle. The CR decreases total skeletal muscle DAG and ceramide content along with weight loss and improves mitochondrial function in skeletal myocytes [4].

The CR as an intervention has consistently shown to improve insulin sensitivity, reduce oxidative stress and improve tissue functions in virtually all body organs and cardiometabolic outcomes $[5,6]$. Yet despite its clear benefits, CR may seem difficult to implement as a long-term therapy in obese patients for multiple reasons, like dietary adherence, perceived decreases in quality of life [7]. Understanding the mechanisms by which CR improves cardiometabolic fitness is of immense value as it would illuminate novel therapeutic targets and open up better treatment strategies [8].

\section{Concept of redox balance and thioredoxin (txn) system}

The cellular redox (reduction/oxidation) balance is regulated by the activity of several antioxidant systems including thioredoxin (TXN), a thiol oxidoreductase system that comprises of TXN, TXN reductase and NADPH [9]. TXN plays an important role in maintaining cellular redox status [10]. Oxidized proteins generated by exposure to reactive oxygen species (ROS) are reduced by the oxidoreductase activity of TXN. Oxidized TXN generated in the process is then reduced and brought back to normal form by NADPH-dependent TXN reductase. The thioredoxin interacting protein (TXNIP), an $\alpha$-arrestin protein, negatively modulates the activity of TXN and influences the cellular redox balance [11]. In addition, genetic and epigenetic variations in TXNIP have been associated with chronic metabolic disorders such as diabetes, MetS and hypertension [12]. TXNIP is expressed in all metabolically important tissues like liver, adipose and skeletal muscle, and is a key player in the regulation of glucose homeostasis and lipid metabolism in peripheral tissues. The expression of TXNIP is upregulated by glucose and repressed by insulin level in skeletal muscle and cultured adipocytes. Further, the expression of TXNIP is elevated in the skeletal muscle and adipose tissue of MetS and T2DM subjects.

The TXN system represents the key regulator mechanism of insulin-stimulated glucose transport in skeletal muscle and involved in the metabolic disruption and IR [13]. Further, the improvement in metabolic instability and insulin sensitivity brought about by CR has been linked to the TXN system. The TXNIP plays a key negative regulator of insulin-stimulated glucose uptake and in cellular fuel substrate partitioning in skeletal muscle $[14,15]$. Being a key regulator of glucose and lipid metabolism, TXNIP influences metabolic regulation through multiple actions including insulin release from $\beta$-cells, glucose production from liver and glucose uptake from peripheral tissues like muscle and adipose $[16,17]$. TXNIP also affects the general metabolism by acting as a nutrient sensor in discrete regions of brain and playing a role in the regulation of fuel utilization and energy expenditure $[18,19]$.

\section{Mechanism for CR to Improve Insulin Sensitivity and IR}

\section{CR and TXN system}

For regulating glucose metabolism, TXNIP binds and negatively regulates thioredoxin via a di-sulphide bond and inhibits glucose uptake, the effect which is overcome by insulin [20]. TXNIP negatively regulates protein kinase $\mathrm{B}$ (PKB), also known as Akt and is a serine/threonine-specific protein kinase playing a key role in multiple cellular processes such as glucose metabolism, apoptosis, cell proliferation, transcription and cell migration. TXNIP also regulates glucose uptake, by maintaining PTEN (Phosphatase and Tensin) homolog protein in a reductive, which is the active state. There is documented evidence that the downregulation of TXNIP plays a role in the insulin-sensitizing effects of CR in the muscle [2]. Further, the global redox status (e.g., reduced/oxidized glutathione) of the skeletal muscle may be the important trigger for induction of TXNIP [21]. But, various factors associated with muscle insulin sensitivity (e.g., mitochondrial respiration/ROS, ceramide, diacylglycerol) do not change with CR. The levels of carnosine, anserine, and taurine in the skeletal muscle increase with CR, which are potent scavengers of lipid- and sugar-derived reactive carbonyl species (RCS). There is a causal role for RCS in obesity-related cardiometabolic diseases [22]. Autophagy is another process which enhance cellular metabolic fitness in response to CR (Figure 2). 


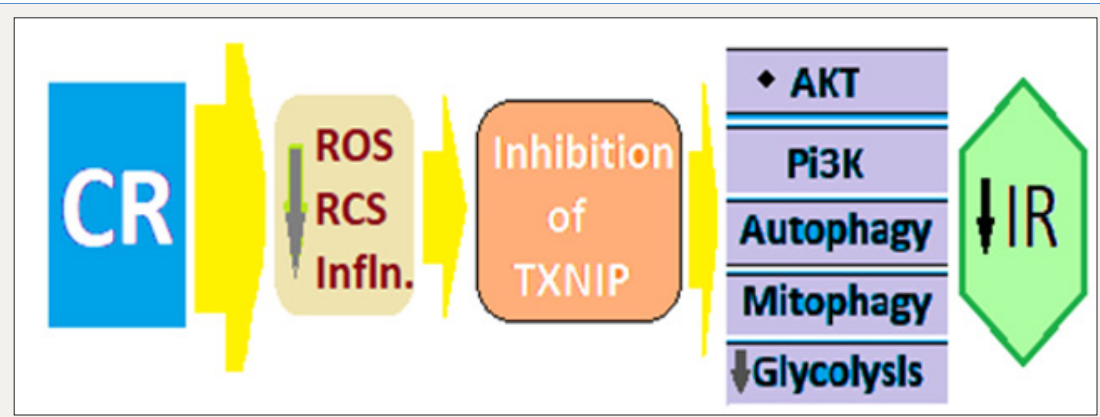

Figure 2: Possible mechanism for CR to improves skeletal muscle insulin sensitivity via TXNIP downregulation.

In the animal studies the TXNIP-deficient mice exhibit hypoglycemia during prolonged fasting, maintain skeletal muscle insulin sensitivity when challenged with a high-fat diet, and are unable to utilize lipid fuels. The high levels of TXNIP in vitro studies decrease insulin-stimulated glucose transport and elevate cellular oxidative stress. Further, insulin-resistant individuals and those with T2DM exhibit elevations in TXNIP mRNA [23].

The downregulation of TXNIP plays a role in the insulinsensitizing effects of $\mathrm{CR}$ in the muscle. In response to $\mathrm{CR}$ and subsequent decrease in adipose mass, oxidative stress and inflammation, there occurs downregulation of TXNIP in the skeletal muscle, which eliminates its inhibitory effect on glycolysis and insulin-stimulated Akt activation, glucose transporters, insulin receptor, insulin receptor substrate and phosphatidylinositol 3-kinase (PI3K).

\section{The skeletal mitochondrial function}

There occurs reduced oxidative capacity in skeletal muscle linked to accumulation of intramyocellular triglyceride (IMTG) and IR, both of which affect the mitochondrial function. Paradoxically, the markers of insulin sensitivity improve following CR, but mitochondrial function and IMTG remain unchanged [24]. There may not be a direct relationship between mitochondrial function and IR in obesity. Both, the skeletal muscle insulin resistance and reduced mitochondrial capacity have been proposed to be affected by aging. Exercise increases both mitochondria content and mitochondrial electron transport chain and fatty acid oxidation enzyme activities within skeletal muscle. It appears, though, that CR-induced weight loss does not bear a direct relationship [25].

The skeletal muscle mitochondria have been implicated in IR associated with obesity and T2DM as well as impaired muscle function with normal aging. Further, there is decreased mitochondrial capacity associated with obesity, T2DM and aging. The potential to improve mitochondrial content and function is shown by increasing physical activity, but not by simply weight loss [26]. Weight loss by CR improves insulin sensitivity and decreases the triglyceride content in skeletal muscle [27]. Further, the excess triglyceride storage within skeletal muscle is due to apparent defects in fatty acid metabolism associated with the mitochondria leading to diminished use of fatty acids and increased esterification and storage of lipid within skeletal muscle, leading to accumulation of triglyceride in skeletal muscles and IR.
Experimentally, the relationship between skeletal muscle mitochondrial dysfunction, oxidative stress and insulin resistance in mouse models treated systemically with an antioxidant normalized skeletal muscle mitochondrial function but failed to normalize glucose tolerance and insulin sensitivity. In the evolution of diet-induced obesity and IR, divergent mitochondrial adaptations modulate oxidative stress and energy expenditure without influencing the onset of impaired insulin-mediated glucose uptake [28]. The IR plays a pivotal role in the development of T2DM. With adiposity, there occurs the deposition of lipids in non-adipose tissues when the capacity of adipose tissue is overwhelmed. The hyperlipidaemia-induced ROS production in skeletal muscle promote mitochondrial alterations, lipid accumulation and inhibition of insulin action [29].

Further, during the development of T2DM, skeletal muscle is a major site of IR. This has been linked to mitochondrial dysfunction and impaired fatty acid oxidation. Some hormones like insulin, thyroid hormones and adipokines (e.g., leptin, adiponectin) have positive effects on muscle mitochondrial bioenergetics through their direct or indirect effects on mitochondrial biogenesis, mitochondrial protein expression, mitochondrial enzyme activities and/or AMPK pathway activation, all of which can improve fatty acid oxidation [30].

\section{Mechanism for Txnip to Improve IR and Various Meta- bolic Effects}

\section{Pancreatic $\beta$-cells mass}

There occurs $\beta$-cell loss in T1DM and dysfunction of $\beta$-cells along with IR in T2DM. In these conditions, the islet's TXNIP gene is upregulated. The $\beta$-cells are susceptible to oxidative stress, and cell death due to apoptosis is a key factor in the pathogenesis of both T1DM and T2DM, following glucose induced stimulation of TXNIP expression in islets (Figure 3). In rodent diabetic models, the TXNIP expression is markedly elevated and has a significant impact on the functioning of $\beta$-cells [31]. The TXNIP may be the critical link between glucose toxicity and $\beta$-cell apoptosis and plays a pivotal role in mediating the detrimental effects of elevated glucose on $\beta$-cells. It induces apoptosis of $\beta$-cells primarily by activating the mitochondrial death pathway through the stimulation of apoptosis signal-regulating kinase 1 (ASK1) and involves cytochrome release and caspase- 3 activation. It also promotes $\beta$-cell apoptosis through the increased expression of pro-apoptotic miR-200 and inhibition of Zinc finger E-box-binding homeobox 1 (ZEB1) expression, 
and mediates glucose dependent upregulation of islet amyloid polypeptide.

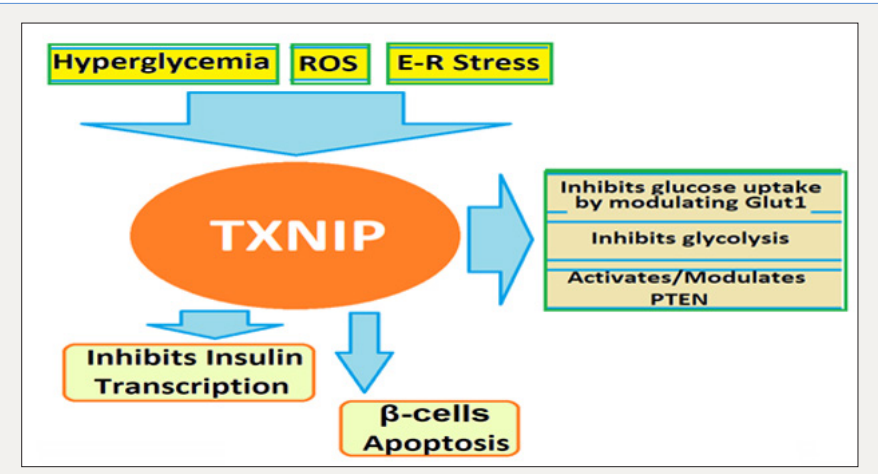

Figure 3: TXNIP associated regulation of glucose metabolism and related projections.

TXNIP regulates of glucose metabolism by inhibiting both its uptake and breakdown by cells. The TXNIP upregulates expression of several miRNAs including miR-204 that mediate the TXNIP induced inhibition of insulin production. MiR-204 has been shown to function through downregulation of MafA, which is a known transcriptional regulator of insulin gene. The expression of miR124a, miR200 and miR-204 are altered in the diabetic condition consistent with the increased expression of TXNIP.

\section{TXNIP controls peripheral insulin sensitivity}

TXNIP is a key component in the improvement of insulin sensitivity in response to $\mathrm{CR}$ in obese subjects. A study involving CR for 16 weeks resulted in marked reduction of TXNIP levels in the skeletal muscle of obese adults, which significantly correlated with the improvement in insulin sensitivity and enhanced non-oxidative disposal of glucose [32]. The TXNIP null mice gain more weight and have higher fat mass, when fed on a high fat diet compared to the WT animals, but in spite of increased adiposity, TXNIP null mice have improved insulin sensitivity and do not develop features of metabolic syndrome [33]. Metformin and Glucagon like peptide-1 (GLP-1) agonists downregulate the expression of TXNIP, which may contribute to their therapeutic efficacy in the treatment of DM [34,35].

\section{TXNIP key regulator for hepatic gluconeogenesis}

TXNIP plays a critical role in hepatic glucose production. The liver specific TXNIP knockout mice suffer from fasting hypoglycemia and elicit a diminished response to glucagon. Hepatocytes isolated from TXNIP deficient animals produce significantly less glucose as compared to hepatocytes obtained from the wild type. TXNIP has been shown to influence cellular redox state and alter NADP/ NADPH and NAD/NADH concentrations, explainable by the changes in glucose production from liver.

\section{TXNIP nutrient sensor and regulator of energy expenditure}

TXNIP is highly expressed in discrete areas of hypothalamus and brainstem having centres for metabolic regulation for metabolic homeostasis and energy balance. Hypothalamic expression of TXNIP is repressed by anorexigenic indicators (refeeding, insulin and leptin) and stimulated during fasting in healthy lean mice. TXNIP expression is elevated in various mouse models of obesity and diabetes and downregulation of hypothalamic TXNIP expression prevents diet-induced obesity and insulin resistance. Thus, hypothalamic TXNIP plays a critical role in nutrient sensing and the regulation of fuel utilization. TXNIP controls multiple aspects of energy metabolism by regulating key processes in adipose, brain, liver, muscle and pancreatic $\beta$-cells.

\section{TXNIP regulates metabolic homeostasis}

TXNIP functions primarily through the inhibition of TXN activity, playing a vital role in maintaining a reducing environment in the cell. Low levels of ROS are important for optimal cellular signaling, but excessive generation of ROS impairs pancreatic cell function and reduces insulin sensitivity in skeletal muscle and adipose [36].

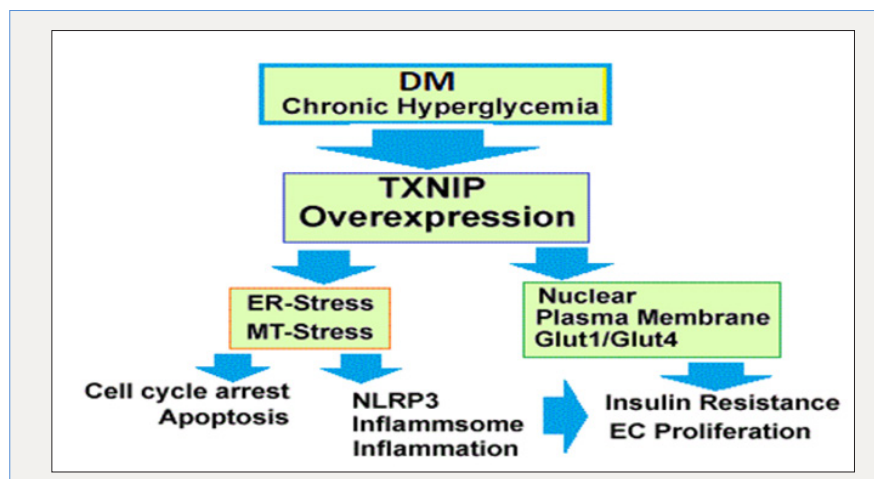

Figure 4: DM and Hyperglycemia upregulate TXNIP, which affects several aspects of $\beta$-cell Function.

TXNIP is also involved in NOD-like receptor Protein-3 (NLRP3) inflammasome activation in a redox dependent manner (Figure 4). NLRs are present in cytoplasm where they act as receptors and detect danger signals emanating from cells undergoing stress, damage or abnormal death as well as by exogenous signals coming from pathogens. TXNIP initiated NLRP3 inflammasome activation has been implicated in obesity-induced insulin resistance and beta cell failure [37]. TXNIP inhibits cellular glucose uptake by regulating the expression and modulating the localization of glucose transporter (Glut1). TXNIP directly interacts with Glut1, reducing its plasma membrane expression by facilitating its endocytosis [38]. In addition, TXNIP also inhibits glucose uptake by inhibiting glycolysis and increasing oxidative metabolism of pyruvate [39]. TXNIP inhibits glycolysis by downregulating the expression of key glycolytic enzymes through the inhibition of hypoxia-induced transcription factor (HIF1 $\alpha$ ) expression. TXNIP also regulates and activates Phosphatase and Tensin homolog (PTEN) lipid phosphatase by a REDOX-sensitive mechanism. PTEN is a natural inhibitor of PI3 kinase/AKT pathway and its activation negatively regulates glucose uptake and metabolism. 
The up-regulation of TXNIP is a short-term response to high calories diet but repeated bouts of over-nutrition lead to a permanent increase in TXNIP expression and accompanying adverse effects. G-protein coupled receptors (GPCRs) are involved in almost all the aspects of metabolic homeostasis. TXNIP has been shown to bind glucose transporter (Glut1) and regulate its endocytosis in TRVb-1 cells and TXNIP may directly interacts with glucose transporters in insulin sensitive tissues like muscle and adipose.

\section{Conclusion-The Additives to Polypharmacy}

\section{CR - an essential additive to therapy}

To endorse CR is of immense value, as it consistently leads to improved cardiometabolic outcomes and exerts beneficial effects on every organ system. CR may involve cutting down the caloric intake by about 40 percent with provision for adequate dietary protein and essential elements like vitamins and minerals. Yet, CR which is simple to conceptualize, is difficult to implement in clinical practice. The hinderance lies in convincing the subjects to follow the rigid dietary programme, its chronic adherence and allaying fear about compromised QOL issues. Still, the focus on CR is important within a specific disease context to clearly delineate underlying mechanisms and exploit the research to achieve therapeutic goals. Finally, understanding the mechanisms by which CR improves cardiometabolic fitness is immensely valuable as it would add to fulfil therapeutic targets and allow to plan better treatment strategies.

\section{TXNIP: A potential therapeutic target}

TXNIP may appear a potential novel therapeutic target. Certain anti-diabetic agents like metformin, GLP-1 agonists and CRMs like resveratrol have been shown to inhibit TXNIP expression. Verapamil - a calcium channel blocker, tranilast - a tryptophan metabolite and allopurinol reduce TXNIP levels in vivo and in vitro studies. Certain calcium channel blockers by lowering TXNIP levels protect $\beta$-cells from apoptosis. Certain issues are important: First, TXNIP expression is upregulated by glucose and elevated levels of TXNIP are found in diabetic subjects and confirmed by animal models. Second, expression of TXNIP is also regulated by insulin, amino acids and nutritional signals like feeding-fasting, suggesting its importance in the regulation of metabolic homeostasis. Third, overexpression of TXNIP is associated with metabolic abnormalities including apoptosis of pancreatic $\beta$-cells, reduced insulin sensitivity and decreased energy expenditure.

The TXNIP is a significant player in regulating insulin sensitivity of peripheral tissues and its overexpression causes reduced insulin sensitivity. The deficiency of TXNIP appears beneficial and animals lacking TXNIP have normal insulin sensitivity and do not develop diabetes or other metabolic abnormalities. Anti-diabetic agents like Insulin and metformin, and CRMs like resveratrol have all been shown to inhibit TXNIP expression. Metformin and CR mimetics like resveratrol are known activator of AMPK, which in turn inhibits expression of TXNIP mRNA and augments degradation of TXNIP protein. GLP-1 receptor agonists regulate expression of
TXNIP by accelerating its proteosomal degradation in a cAMP/PKA dependent manner.

The TXNIP based therapeutic approach may achieve the requirement of a perfect anti-diabetic therapy by not only improving insulin secretion and sensitivity but also ameliorating global pathology of diabetes, including cardiovascular and microvascular complications. But, on a cautious note, the loss of TXNIP with the use of TXNIP inhibitors may have serious consequences as TXNIP expression is required for maintaining normal fasting glycaemia [40]. Secondly, TXNIP being a tumour suppressor, its loss may be associated with increased incidence of cancer. Therefore, use of TXNIP inhibitors could potentially be associated with an elevated risk of hypoglycaemic episodes and increased incidence of cancer.

\section{References}

1. Kelley DE, He J, Menshikova EV (2002) Dysfunction of mitochondria in human skeletal muscle in type 2 diabetes. Diabetes 51(10): 2944-2950.

2. Johnson ML, Distelmaier K, Lanza IR (2016) Mechanism by which caloric restriction improves insulin sensitivity in sedentary obese adults. Diabetes 65(1): 74-84.

3. Toledo FG, Menshikova EV, Azuma K (2008) Mitochondrial capacity in skeletal muscle is not stimulated by weight loss despite increases in insulin action and decreases in intramyocellular lipid content. Diabetes 57(4): 987-994.

4. Dubé JJ, Amati F, Toledo FG (2011) Effects of weight loss and exercise on insulin resistance, and intramyocellular triacylglycerol, diacylglycerol and ceramide. Diabetologia 54(5): 1147-1156.

5. Barnosky AR, Hoddy KK, Unterman TG (2014) Intermittent fasting vs daily calorie restriction for type 2 diabetes prevention: a review of human findings. Transl Res 164(4): 302-311.

6. Yoshimura E, Kumahara H, Tobina T (2014) Lifestyle intervention involving calorie restriction with or without aerobic exercise training improves liver fat in adults with visceral adiposity. J Obes doi: $10.1155 / 2014 / 197216$.

7. Bales CW, Kraus WE (2013) Caloric restriction: implications for human cardiometabolic health. J Cardiopulm Rehabil Prev 33(4): 201-208.

8. Anderson EA (2016) Cutting calories and TXNIP from the skeletal muscle to restore insulin sensitivity. Diabetes 65(1): 16-18.

9. Lu J, Holmgren A (2014) The thioredoxin antioxidant system. Free Radic Biol Med 66: 75-87.

10. Nordberg J, Arner ES (2001) Reactive oxygen species, antioxidants, and the mammalian thioredoxin system. Free Radic Biol Med 31(11): 12871312.

11. Alhawiti NM, Al Mahri SA, Aziz MA (2017) TXNIP in metabolic regulation: Physiological role and therapeutic outlook. Curr Drug Targets 18(9): 1095-1103.

12. Ferreira NE, Omae S, Pereira A (2012) Thioredoxin interacting protein genetic variation is associated with diabetes and hypertension in the Brazilian general population. Atherosclerosis 221(1): 131-136.

13. Hui ST, Andres AM, Miller AK (2008) TXNIP balances metabolic and growth signaling via PTEN disulfide reduction. Proc Natl Acad Sci USA 105(10): 3921-3926.

14. Chutkow WA, Birkenfeld AL, Brown JD (2010) Deletion of the alphaarrestin protein Txnip in mice promotes adiposity and adipogenesis while preserving insulin sensitivity. Diabetes 59(6): 1424-1434.

15. De Balsi KL, Wong KE, Koves TR (2014) Targeted metabolomics connects thioredoxin-interacting protein (TXNIP) to mitochondrial fuel 
selection and regulation of specific oxidoreductase enzymes in skeletal muscle. J Biol Chem 289(12): 8106-8120.

16. Shalev A (2014) Minireview: Thioredoxin-interacting protein: regulation and function in the pancreatic beta-cell. Mol Endocrinol 28(8): 1211-1220.

17. Oka S, Yoshihara E, Bizen-Abe A (2009) Thioredoxin binding protein-2/ thioredoxin-interacting protein is a critical regulator of insulin secretion and peroxisome proliferator-activated receptor function Endocrinology 150(3): 1225-1234.

18. Blouet C, Liu SM, Jo YH (2012) TXNIP in Agrp neurons regulates adiposity, energy expenditure, and central leptin sensitivity. J Neurosci 32(29): 9870-9877.

19. Blouet C, Schwartz GJ (2011) Nutrient-sensing hypothalamic TXNIP links nutrient excess to energy imbalance in mice. J Neurosci 31(16): 6019-6027.

20. Parikh H, Carlsson E, Chutkow WA (2007) TXNIP regulates peripheral glucose metabolism in humans. PLoS Med 4(5): e158.

21. Schulze PC, Yoshioka J, Takahashi T (2004) Hyperglycemia promotes oxidative stress through inhibition of thioredoxin function by thioredoxin-interacting protein. J Biol Chem 279(29): 30369-30374.

22. Curtis JM, Grimsrud PA, Wright WS (2010) Downregulation of adipose glutathione S-transferase A4 leads to increased protein carbonylation, oxidative stress, and mitochondrial dysfunction. Diabetes 59(5): 11321142.

23. Hui TY, Sheth SS, Diffley JM (2004) Mice lacking thioredoxin-interacting protein provide evidence linking cellular redox state to appropriate response to nutritional signals. J Biol Chem 279(23): 24387-24393.

24. Rabøl R, Svendsen PF, Skovbro M (2009) Reduced skeletal muscle mitochondrial respiration and improved glucose metabolism in nondiabetic obese women during a very low-calorie dietary intervention leading to rapid weight loss. Metabolism 58(8): 1145-1152.

25. Menshikova EV, Ritov VB, Dube JJ (2017) Calorie restriction-induced weight loss and exercise have differential effects on skeletal muscle mitochondria despite similar effects on insulin sensitivity. J Gerontol A Biol Sci Med Sci 73(1): 81-87.

26. Toledo FG, Goodpaster BH (2013) The role of weight loss and exercise in correcting skeletal muscle mitochondrial abnormalities in obesity, diabetes and aging. Mol Cell Endocrinol 379(1-2): 30-34.

27. Kelley DE, Goodpaster BH (2001) Skeletal muscle triglyceride. An aspect of regional adiposity and insulin resistance. Diabetes Care 24(5) 933-941.
28. Boudina S, Sena S, Sloan C (2012) Early mitochondrial adaptations in skeletal muscle to diet-induced obesity are strain dependent and determine oxidative stress and energy expenditure but not insulin sensitivity. Endocrinology 153(6): 2677-2688

29. Di Meo S, Iossa S, Venditti P (2017) Skeletal muscle insulin resistance: role of mitochondria and other ROS sources. J Endocrinol 233(1): R15-R42.

30. Aguer C, Harper ME (2012) Skeletal muscle mitochondrial energetics in obesity and type 2 diabetes mellitus: endocrine aspects. Best Pract Res Clin Endocrinol Metab 26(6): 805-819.

31. Chen J, Saxena G, Mungrue IN (2008) Thioredoxin-interacting protein: a critical link between glucose toxicity and beta-cell apoptosis. Diabetes 57(4): 938-944.

32. Anderson EJ (2016) Cutting calories and TXNIP from the skeletal muscle to restore insulin sensitivity. Diabetes 65(1): 16-18.

33. Chutkow WA, Birkenfeld AL, Brown JD (2010) Deletion of the alphaarrestin protein Txnip in mice promotes adiposity and adipogenesis while preserving insulin sensitivity. Diabetes 59(6): 1424-1434.

34. Chong CR, Chan WP, Nguyen TH (2014) Thioredoxin-interacting protein: pathophysiology and emerging pharmacotherapeutics in cardiovascular disease and diabetes. Cardiovasc Drugs Ther 28(4): 347-360.

35. Chai TF, Hong SY, He H (2012) A potential mechanism of metforminmediated regulation of glucose homeostasis: inhibition of Thioredoxininteracting protein (TXNIP) gene expression. Cell Signal 24(8): 17001705.

36. Nishikawa T, Araki E (2007) Impact of mitochondrial ROS production in the pathogenesis of diabetes mellitus and its complications. Antioxid Redox Signal 9(3): 343-353.

37. Mori MA, Bezy O, Kahn CR (2011) Metabolic syndrome: is Nlrp3 inflammasome a trigger or a target of insulin resistance? Circ Res 108(10): 1160-1162.

38. Wu N, Zheng B, Shaywitz A (2013) AMPK-dependent degradation of TXNIP upon energy stress leads to enhanced glucose uptake via GLUT1. Mol Cell 49(6): 1167-1175.

39. Yu FX, Chai TF, He H (2010) Thioredoxin-interacting protein (Txnip) gene expression: sensing oxidative phosphorylation status and glycolytic rate. J Biol Chem 285(33): 25822-25830.

40. Patwari P, Chutkow WA, Cummings K (2009) Thioredoxin-independent regulation of metabolism by the alpha-arrestin proteins. J Biol Chem 284(37): 24996-25003.

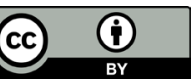

Creative Commons Attribution 4.0 International License

For possible submissions Click Here

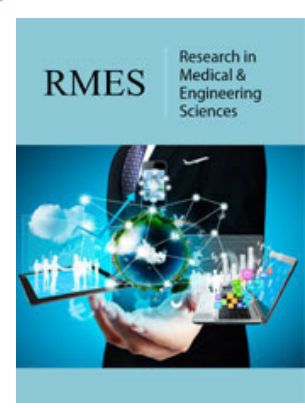

\section{Research in Medical \& Engineering Sciences}

\section{Benefits of Publishing with us}

- High-level peer review and editorial services

- Freely accessible online immediately upon publication

- Authors retain the copyright to their work

- Licensing it under a Creative Commons license

- Visibility through different online platforms 